\title{
Food Defense - do conceito às atuais exigências do mercado internacional
}

\author{
Food Defense - from the concept to current demands of international market \\ Food Defense - conozca el concepto y los requisitos del mercado internacional
}

Recebido: 01/12/2021 | Revisado: 09/12/2021 | Aceito: 17/12/2021 | Publicado: 24/12/2021

\author{
Elaine Leão Inácio de Melo Andrade \\ ORCID: https://orcid.org/0000-0001-7281-292X \\ Universidade Federal do Estado do Rio de Janeiro, Brasil \\ E-mail: elaine.leao@edu.unirio.br \\ Gilberto Carvalho de Oliveira \\ ORCID: https://orcid.org/0000-0001-6713-1126 \\ Universidade Federal do Rio de Janeiro, Brasil \\ E-mail: gilbertooliv@gmail.com \\ Otniel Freitas-Silva \\ ORCID: https://orcid.org/0000-0002-7658-8010 \\ Embrapa Agroindústria de Alimentos, Brasil \\ E-mail: otniel.freitas@embrapa.br
}

\begin{abstract}
Resumo
O conceito food defense emergiu nos EUA como resposta aos atentados terroristas do 11 de Setembro, em 2001. A Food and Drug Administration (FDA) e outras agências desenvolveram medidas para proteger a cadeia alimentar do país aos ataques maliciosos. O objetivo consistia em adotar medidas preventivas da contaminação intencional da água e alimentos, onde indivíduos recorrem a agentes (biológicos, químicos ou físicos) com o intuito de causar prejuízos às organizações, governos ou à população. A vulnerabilidade da cadeia alimentar propiciou a difusão deste conceito através de normas certificáveis como: International Featured Standards (IFS), British Retail Consortium (BRC) e Food Safety Systems Certification (FSSC 22000). Este estudo tem como objetivo a emergência do conceito food defense (defesa alimentar), junto aos termos já conhecidos: food quality (qualidade alimentar), food safety (segurança alimentar), food security (acessibilidade alimentar) e food fraud (fraude alimentar), através das regulamentações, leis, metodologias e ferramentas para implementação de food defense nas cadeias de produção e distribuição de alimentos de empresas exportadoras até empresas familiares. Metodologia: análise qualitativa através de pesquisa bibliográfica nas bases Scopus, Web of Science e SciELO. Espera-se que os resultados deste estudo despertem a necessidade de formar profissionais da área de alimentos conhecedores do conceito food defense, e que orientem a adoção de medidas conceituais e práticas que previnam e controlem a contaminação intencional de alimentos, de forma a atender às exigências dos mercados consumidores e às normas e legislações vigentes pertinentes à qualidade e segurança dos alimentos.
\end{abstract}

Palavras-chave: Defesa alimentar; Fraude alimentar; Segurança alimentar; Bioterrorismo.

\begin{abstract}
The food defense concept emerged in the US in response to the terrorist attacks of September 11, 2001. Thus, the Food and Drug Administration (FDA) and other agencies developed measures to protect the country's food chain from malicious attacks. The objective was to adopt preventive measures against the intentional contamination of water and food, where individuals resort to agents (biological, chemical, or physical) to harm organizations, governments or the population. The vulnerability of the food chain enabled the dissemination of this concept through certifiable standards such as: International Featured Standards (IFS), British Retail Consortium (BRC) and Food Safety Systems Certification (FSSC 22000). This study aims at the emergence of the food defense concept, along with the already known terms: food quality, food safety, food security and food fraud, through regulations, laws, methodologies, and tools for implementing food defense in food production and distribution chains from exporting companies to family businesses. Methodology: qualitative analysis through bibliographic research in Scopus, Web of Science and SciELO databases. Conclusion: it is expected that the results of this study arouse the need for training professionals in the food area, including concepts such as food defense, and that guide the adoption of measures in the conceptual and practical spheres that prevent and control the intentional contamination of food, from to meet the constant demands of the consumer markets and the adoption of norms and legislation in force regarding the quality and safety of food.
\end{abstract}

Keywords: Food defense; Food fraud; Food safety; Bioterrorism.

\section{Resumen}

El concepto de food defense surgió en los EUA en respuesta a los ataques terroristas del 11 de septiembre de 2001. La Administración de Alimentos y Medicamentos (FDA) y otras agencias han desarrollado medidas para proteger la 
cadena alimentaria del país de ataques maliciosos. El objetivo fue adoptar medidas preventivas contra la contaminación intencional de agua y alimentos, donde los individuos recurren a agentes (biológicos, químicos o físicos) con el fin de dañar a organizaciones, gobiernos o la población. La vulnerabilidad de la cadena alimentaria permitió la difusión de este concepto a través de estándares certificables como: International Featured Standards (IFS), British Retail Consortium (BRC) y Food Safety Systems Certification (FSSC 22000). Este estudio tiene como objetivo el surgimiento del concepto de defensa alimentaria, junto con los términos ya conocidos: calidad alimentaria, inocuidad alimentaria, seguridad alimentaria y fraude alimentario. A través de normativas, leyes, metodologías y herramientas para implementar la defensa alimentaria en las cadenas de producción y distribución de alimentos de empresas exportadoras a empresas familiares. Metodología: análisis cualitativo mediante investigación bibliográfica en las bases de datos Scopus, Web of Science y SciELO. Se espera que los resultados de este estudio susciten la necesidad de formar profesionales de la alimentación familiarizados con el concepto de defensa alimentaria, y que orienten la adopción de medidas conceptuales y prácticas que prevengan y controlen la contaminación intencional de los alimentos, con el fin de atender las demandas de los mercados consumidores y las normas y legislaciones vigentes en materia de calidad e inocuidad de los alimentos.

Palabras clave: Defensa alimentaria; Fraude alimentario; Seguridad alimenticia; Bioterrorismo.

\section{Introdução}

O sistema alimentar continua a aumentar em complexidade, diversidade e interdependência entre sistemas domésticos e globais. O desenvolvimento de sistemas abrangentes de gestão de risco para proteger o abastecimento de alimentos, estabelece uma base para minimizar os impactos econômicos e na saúde pública, promovendo segurança alimentar. De acordo com a Food Safety and Inspection Service (FSIS), a responsabilidade pela cadeia de abastecimento alimentar global é compartilhada por todos os níveis do governo (estrangeiro e doméstico) e por meio de parcerias público-privadas colaborativas com a indústria (Agriculture, 2021).

Alguns dias após os eventos terroristas de 11 de setembro de 2001 nos Estados Unidos, identificou-se a disseminação deliberada de esporos de antraz potencialmente letais em cartas enviadas através do Serviço Postal dos Estados Unidos acrescentando a liberação deliberada de agentes biológicos às realidades da vida no século XXI. Os esporos do antraz foram encontrados em quatro envelopes, cinco pessoas morreram contaminadas, o ataque com antraz causou uma grande interrupção dos serviços postais em muitos países do mundo e teve enormes consequências econômicas, de saúde pública e de segurança. Isso despertou preocupações internacionais renovadas sobre o bioterrorismo, provocando contramedidas em muitos países, inclusive no que diz respeito a contaminações de alimentos e água (World Health Organization, 2007).

As doenças de origem alimentar são causadas por mais de 200 diferentes patógenos (agentes que podem causar doenças), dos quais temos conhecimento atualmente. Isso inclui vírus, bactérias, parasitas e toxinas, além de um grande número de contaminantes químicos e metais potenciais. A variedade de agentes associados às doenças transmitidas por alimentos tem crescido constantemente nas últimas décadas, e é provável que essa lista continue a aumentar. O surgimento de novos patógenos de origem alimentar requer tecnologias atualizadas que possam detectar a presença de novos agentes em uma variedade de alimentos. Abordar esses riscos emergentes requer cooperação entre indústria, academia e governo para compartilhar informações e estabelecer protocolos de teste (U.S. Food and Drug Administration, 2007).

As crescentes preocupações conduziram à emergência de novos conceitos e metodologias como o food defense, que Segundo Severino \& Almeida (2016), é um conceito que emergiu com a abordagem de proteger, prevenir e mensurar o impacto sobre a população e a indústria produtora de alimentos de um ataque de contaminação intencional.

A contaminação intencional da cadeia produtiva de alimentos tem o potencial de ocasionar efeitos desastrosos e de longo alcance, incluindo morbidade direta e/ou mortalidade, interrupção da distribuição de alimentos, perda da confiança do consumidor no governo e no fornecimento de alimentos, insucesso e restrições comerciais e graves efeitos sobre a economia (Busta \& Kennedy, 2011).

Desde 1998, os seguintes agentes foram identificados como envolvidos em eventos de contaminação deliberada: arsênico, cianeto, fezes, herbicida, limpador doméstico, inseticida, sulfato de nicotina, pesticida, veneno de rato, tetramina e 
tálio. Os países que experimentaram eventos de contaminação intencional incluem Austrália, Canadá, China / Hong Kong, Iraque, Itália, Japão, Coréia, Filipinas, Taiwan, Tailândia e os EUA (Dalziel, 2009).

Este artigo tem como ponto de partida, o estudo epistemológico do uso de agentes utilizados pelo homem abordando os conceitos de guerra biológica, bioterrorismo, pandemia, biossegurança, com a finalidade de contribuir com pressupostos para compreensão do conceito de food defense. Seguida de uma abordagem conceitual de forma a esclarecer o conceito de food defense (defesa alimentar), ressaltando sua diferença dos termos: food quality (qualidade dos alimentos), food safety (segurança dos alimentos) e food fraud (fraude alimentar), e finalizando com a elucidação de requisitos de food defense presentes nos referenciais reconhecidos pela Global Food Safety Initiative (GFSI) como a International Featured Standards (IFS), British Retail Consortium (BRC) e Food Safety Systems Certification (FSSC 22000) além de novas metodologias que ressaltam a importância da implantação de um plano de food defense.

\section{Metodologia}

Do ponto de vista metodológico, este artigo foi desenvolvido a partir de uma pesquisa exploratória, que de acordo com Rodrigues (2007), "busca se familiarizar com os fenômenos surgidos durante a pesquisa, explorando os próximos passos mais profundamente e com maior precisão" baseada em fontes bibliográficas secundárias e fontes documentais primárias sobre o tema, incluindo diretrizes governamentais, legislações, regulamentações e sistemas relacionados às iniciativas de food defense principalmente nos EUA, mas também em estados europeus, como o Reino Unido, e Agências Especializadas Internacionais, como a Organização Mundial da Saúde (OMS), a agência federal do Departamento de Saúde e Serviços Humanos dos Estados Unidos (FDA) e o Departamento de Agricultura dos Estados Unidos (USDA).

Segundo as suas origens, as fontes da pesquisa bibliográfica podem ser primárias, secundárias e terciárias. Para Albrecht e Ohira (2000):

Fontes primárias - são as mais importantes, por representarem a grande produção técnica e científica da área. Nelas incluem-se: os livros, os periódicos e publicações seriadas, os anais de eventos, os relatórios técnicos, as normas técnicas, as teses e dissertações e as patentes.

Fontes secundárias - são as que organizam, sob a forma de índices e resumos, as informações de fontes primárias, facilitando assim o conhecimento e o acesso às mesmas. As publicações englobadas nesta categoria, normalmente são designadas como "obras de referência".

Fontes terciárias - são as que orientam o usuário para a utilização das fontes secundárias e primárias, facilitando localização e o acesso às informações. Elas representam o ponto de partida para as ações da coleta (Albrecht \& Ohira, 2000, p139-140).

Nesta revisão foram utilizadas ferramentas de investigação que passaram pela utilização da internet, documentos online, artigos, revistas e livros. A pesquisa dos artigos foi realizada nas bases Scopus, Web of Science e SciELO, entre os meses de janeiro de 2020 e outubro de 2021, utilizando os descritores em inglês TITLE-ABS-KEY "food defense" AND "food safety". Foram incluídos artigos em inglês, espanhol e português, limitando-se as seguintes subáreas: agricultura, química, saúde, engenharia, economia, veterinária e negócios, excluindo as demais subáreas. Além disso, para a leitura e seleção dos artigos foi criado um acervo no gerenciador de referências Mendeley Desktop (Version1.16.1, MendeleyLtd., ElsevierInc., NY, USA). Os artigos foram selecionados de acordo com sua relevância, através da leitura dos resumos e análise detalhada dos mesmos de acordo com os critérios de inclusão e exclusão escolhidos. Os critérios de inclusão foram os trabalhos completos com disponibilidade na íntegra e com limite de temporalidade entre o período de 2001 (ano do atentado terrorista às torres gêmeas) a 2021. Em contrapartida os critérios de exclusão foram os estudos que não se referiam estritamente ao tema, os quais não estivessem ligados ao tema principal abordado e artigos duplicados. Após a seleção para a síntese deste trabalho, foi realizado download de todos os artigos escolhidos e prosseguido com a leitura completa dos mesmos na íntegra para a revisão 
de literatura. Dessa forma, contribuindo para o processo de síntese dos resultados de vários estudos, criando um corpo de literatura compreensível.

\section{Resultados e Discussão}

\section{O uso de agentes biológicos como armas}

Vários fatores podem interferir no aumento das contaminações através dos alimentos e de outros vetores, resultando na emergência ou reemergência de doenças. Luna (2002) propõe sete grandes grupos: fatores demográficos, fatores sociais e políticos, fatores econômicos, fatores ambientais, fatores relacionados ao desempenho do setor saúde, fatores relacionados às mudanças e adaptação e manipulação de microrganismos com vistas ao desenvolvimento de armas biológicas, embora, na grande maioria das situações, múltiplos fatores atuam simultaneamente para o surgimento das doenças.

De acordo com Baldy (1991) as doenças transmissíveis, também conhecidas como doenças infecciosas e parasitárias, são doenças causadas por agentes específicos, ou seus produtos metabólicos (toxinas), que resultam da transferência deste agente ou seus produtos, de forma direta ou indireta, de um reservatório para um hospedeiro suscetível, humano ou animal, por meio de um hospedeiro intermediário, de natureza vegetal ou animal, de um vetor ou do meio ambiente. A contaminação é o termo utilizado para indicar a presença de agentes biológicos ou de seus produtos na água, em alimentos, no solo, em objetos ou, transitoriamente, na superfície corpórea (nas mãos, por exemplo), sem a ocorrência de colonização ou infecção. É muito comum a intoxicação através de água ou alimentos contaminados, devido à falta de condições de higiene, em muitas localidades onde não existem sistemas de saneamento, ou não existe fiscalização em produtos alimentícios.

No século XIX, com o desenvolvimento dos métodos de isolamento, cultura e identificação de bactérias, os primeiros programas de guerra biológica ocorreram durante a Primeira Guerra Mundial, na Alemanha e na França. Davison \& Neil (2005) ainda afirmam que esses avanços possibilitaram o desenvolvimento de programas mais elaborados de armas biológicas com maior potencial de destruição, as quais posteriormente passaram a ser conhecidas como armas de destruição em massa, por possuírem baixos custos de produção, fácil acesso a uma ampla gama de agentes biológicos produtores de doenças, transporte fácil de um lugar para outro e dificuldade de detecção por sistemas de segurança de rotina.

Schatzmayr (2013) descreve os parâmetros ideais para um agente ser utilizado como arma biológica: o agente deve ser capaz de exercer determinado efeito de maneira consistente; a dose necessária para produzir o efeito deve ser baixa; o período de incubação deve ser curto e bem definido; a população-alvo não deve ser imune ao agente; o tratamento não deve estar disponível; ter a possibilidade de produzir o agente em grandes quantidades; o agente deve ser disseminado de maneira eficiente; o agente deve ser estável, permitindo sua guarda e seu transporte para as áreas de atuação.

Este levantamento epistemológico sobre agentes biológicos causadores de doenças, tem o objetivo de embasar historicamente conhecimentos que emergem e direcionam conceitos mais recentes especificamente na área de alimentos, como o conceito de food defense. Pode-se perceber a correlação conceitual com o terrorismo alimentar, que de acordo com o WHO (2008) é definido como o ato ou ameaça de contaminação deliberada de gêneros alimentícios para consumo humano ou animal através de agentes químicos, biológicos, radioativos ou nucleares, com o objetivo de causar danos ou morte ou de alterar a ordem social, econômica ou política estabelecidas. Severino (2016) ressalta a similaridade ao bioterrorismo que é definido quando ocorre a libertação intencional de agentes biológicos ou das suas toxinas para provocar doença ou morte em pessoas, animais ou outros, onde estes agentes podem ser provenientes de fontes naturais ou sofrerem alterações pelo homem, para incrementar a sua capacidade de causar doença, tornando-os mais resistentes aos medicamentos existentes ou melhorando a sua capacidade de se disseminarem no meio ambiente.

Segundo a OMS, o combate ao terrorismo alimentar é conseguido através de medidas articuladas de prevenção, detecção, preparação, resposta e comunicação entre os Estados, instituições e pessoas. Para que tais medidas sejam 
concretizadas com sucesso é fundamental que os países tenham previamente implementado programas de segurança consistentes e eficazes (Severino \& Almeida, 2016).

\section{Como surgiu o conceito food defense}

$\mathrm{O}$ ataque às torres gêmeas de 11 de setembro de 2001 seguido da disseminação deliberada de esporos de antraz potencialmente letais em cartas enviadas através do Serviço Postal dos Estados Unidos, obrigaram os Estados Unidos a terem uma visão mais ampla sobre os aspectos de proteção e segurança. Embora a preocupação com a segurança das viagens aéreas passasse a ser o centro das atenções, o governo dos EUA não ignorou as preocupações sobre a segurança do abastecimento alimentar americano. Importantes posições do governo federal questionaram a facilidade com que as organizações terroristas poderiam contaminar partes dos sistemas de abastecimento e distribuição de alimentos (Pear, 2004).

No final de 2001, o FDA e o USDA procuraram determinar o estado de prontidão do sistema alimentar americano contra um ataque intencional, e surge a primeira lei aprovada em junho de 2002: Public Health Security and Bioterrorism Preparadness and Response Act (Office, 2007), exigindo o registro de todas as indústrias do setor de alimentos que envolvessem fabricação, embalagem e armazenagem, com disposições para a prevenção, preparação e resposta a ataques bioterroristas, incluindo a notificação prévia das importações de alimentos para o território norte-americano (P. Severino \& Almeida, 2016).

\section{Diferentes formas de ameaças e proteção aos alimentos}

Representam perigo à segurança dos alimentos, todos os agentes biológicos, químicos e físicos, ou condição do alimento, com potencial de causar um efeito adverso à saúde. O termo perigo não deve ser confundido com o termo risco. Risco, no contexto de segurança de alimentos, significa uma função da probabilidade de ocorrência de um efeito adverso à saúde e a severidade deste efeito quando há a exposição a um perigo específico. Os perigos podem ser intrínsecos ao próprio alimento ou não, e podem ser adicionados intencionalmente nos alimentos. Estes perigos podem causar distúrbios na saúde pública com consequências sociais e econômicas com diferentes níveis de gravidade (Dias et al., 2010).

De acordo com a matriz representada na Figura 1, verifica-se que os conceitos de defesa dos alimentos (food defense) e fraude alimentar (food fraud) encontram-se associados às práticas intencionais, cujas motivações que conduzem a estas ações podem ser ideológicas ou para ganho econômico, respectivamente e por outro lado, qualidade alimentar (food quality) e segurança dos alimentos (food safety) estão associadas a ações não intencionais, que visam a proteção dos sistemas alimentares contra doenças transmitidas por alimentos de ocorrência natural (Severino \& Almeida, 2016). 
Figura 1. Adulteração de alimentos intencional versus não intencional.

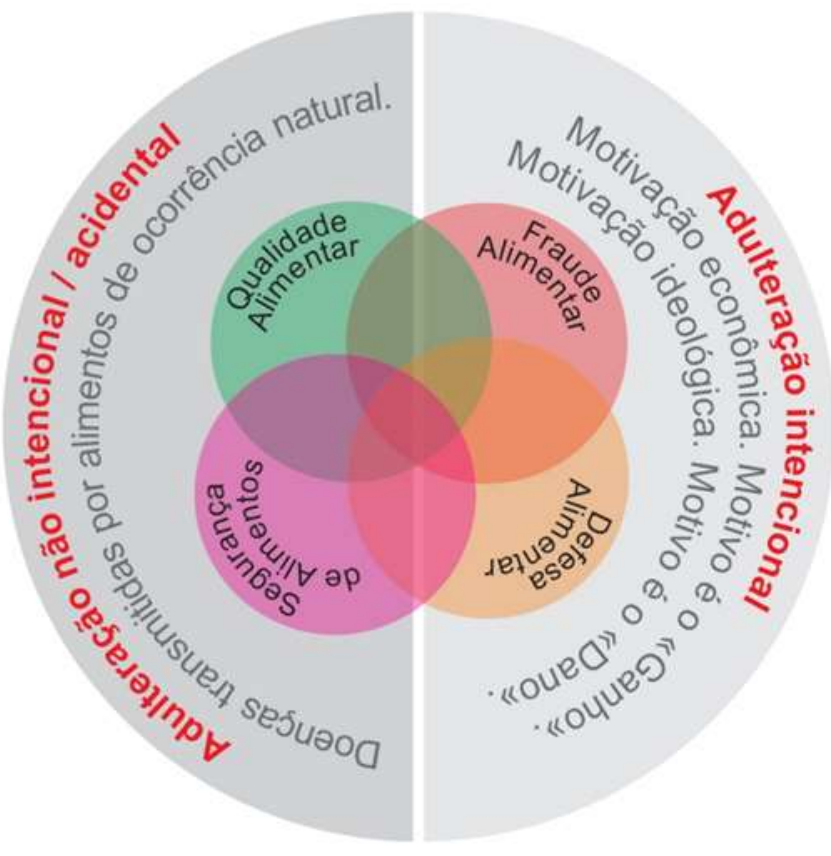

Fonte: Adaptado de FSSC 22000 (2019). ${ }^{\mathrm{a}}$

Para melhor compreensão da matriz, é importante definir os conceitos: food quality, food safety, food fraud, e food defense.

Food quality (qualidade alimentar): O produto deve atender às especificações conforme a categoria do alimento. Este é um ato não intencional (sem intenção de prejudicar). De acordo com a FAO/WHO (2015), food quality inclui todos os atributos que influenciam no valor do produto para o consumidor, tanto atributos negativos: deterioração, contaminação com sujeira, descoloração, odores, como atributos positivos: a origem, cor, sabor, textura e método de processamento do alimento.

O termo food safety (segurança de alimentos), associado à segurança sanitária dos alimentos, refere-se às condições e práticas para preservar a qualidade dos alimentos durante o armazenamento e transporte e centra-se sobre os perigos biológicos, físicos ou químicos, a fim de evitar contaminação e intoxicação alimentar, este é um ato não intencional com perigo à saúde (Severino \& Almeida, 2016).

Food fraud (fraude alimentar) está associada a práticas intencionais assim como food defense. Este termo é definido pela Global Food Safety Initiative (GFSI) como termo coletivo usado para abranger a substituição deliberada e intencional, adição, adulteração ou falsificação de alimentos, ingredientes alimentares ou embalagens de alimentos, rotulagem, informações de produto falsas ou enganosas, declarações feitas sobre um produto para ganho econômico que pode causar impacto na saúde dos consumidores (Spink \& Moyer, 2011).

A definição do GFSI de food defense (defesa alimentar) é: o processo para garantir a segurança dos alimentos, ingredientes, alimentos para animais ou embalagens, de todas as formas de ataque malicioso intencional, incluindo ataque motivado ideologicamente levando a contaminação ou insegurança dos produtos (GFSI BENCHMARKING REQUIREMENTS VERSION 2020, 2020).

A indústria e os reguladores desenvolveram Sistemas de Gestão de Segurança de Alimentos, o APPCC por exemplo, da sigla original em inglês HACCP (Hazard Analisys and Critical Control Points) que é baseado numa série de etapas inerentes ao processamento industrial dos alimentos, incluindo todas as operações que ocorrem desde a obtenção da matéria-prima até o consumo, fundamentando-se na identificação dos perigos potenciais à saúde do consumidor, bem como nas medidas de 
controle das condições que geram os perigos (Ribeiro-Furtini \& Abreu, 2006).

Os princípios de Análise de Perigos e Pontos Críticos de Controle (APPCC), são eficazes contra riscos da segurança de alimentos não intencionais. Embora food defense não seja explicitamente incluída como parte do APPCC, a etapa fundamental de identificação dos perigos físicos, biológicos e químicos aos alimentos convida à inclusão de medidas de ação para impedir a sabotagem alimentar intencional (Klitzke, 2015).

A motivação ou causa raiz para o food defense é a intenção de causar danos ao consumidor ou empresas. Isso é diferente da motivação da food fraud que é exclusivamente para o ganho econômico. No entanto, adulterar a presença de um ingrediente ou substituí-lo por outro de menor valor comercial, pode levar a danos irreversíveis à saúde dos consumidores, ou seja, na perspectiva de obtenção de maiores lucros, este crime prejudicial pode ter um impacto na saúde das populações mais vulneráveis e cruzar fronteiras geográficas. Desta forma, food fraud representa um risco para saúde humana e, portanto, as indústrias devem investir em análises específicas e eficazes de processos produtivos, além de medidas de controle, ações destinadas a reduzir ou eliminar possíveis perigos nos processos que contribuíram para garantir a qualidade e segurança dos produtos (Santos et al., 2020).

Existem ainda outros termos relacionados aos cuidados com os alimentos: food authenticity, food integrity, food protection.

Food authenticity (HM Government, 2014): de acordo com o Elliott Review report, a autenticidade dos alimentos consiste em garantir que os alimentos oferecidos para venda, refiram-se de fato à natureza da substância e a qualidade esperadas pelo comprador. É o estado de não ter sido alterado ou modificado em relação às características esperadas de segurança, qualidade e nutrição. A importância da autenticidade é enfatizada quando o consumidor tem uma questão específica que deve ser respeitada: ser alérgico à algum componente alimentar ou pertencer à grupos religiosos com restrições alimentares, ou simplesmente consumidores com preferências alimentares que não desejam comprar produtos que contenham determinados ingredientes.

Food integrity (Elliott \& Goethem, 2018) : a integridade do alimento é o estado de ser genuíno e indiscutível em sua natureza, genética, origem, identidade, garantindo as expectativas em relação às propriedades do alimento. A integridade dos alimentos é de primordial importância para agregar valor à economia agroalimentar. A food integrity está sob constante ameaça de imitações fraudulentas que tentam explorar esse valor agregado.

Food protection (U.S. Food and Drug Administration, 2007): significa proteger os alimentos contra contaminação não intencional e deliberada. O food protection plan visa implementar uma estratégia de prevenção, intervenção e resposta para aumentar a segurança em todas as etapas da cadeia de suprimento de alimentos.

Juntando os termos food authenticity, food integrity, food protection, aos conceitos apresentados anteriormente: food safety, food quality, food fraud e food defense, pode-se demonstrar a relação entre os termos através da matriz de risco alimentar (Figura 2). 
Figura 2. Matriz de risco alimentar.

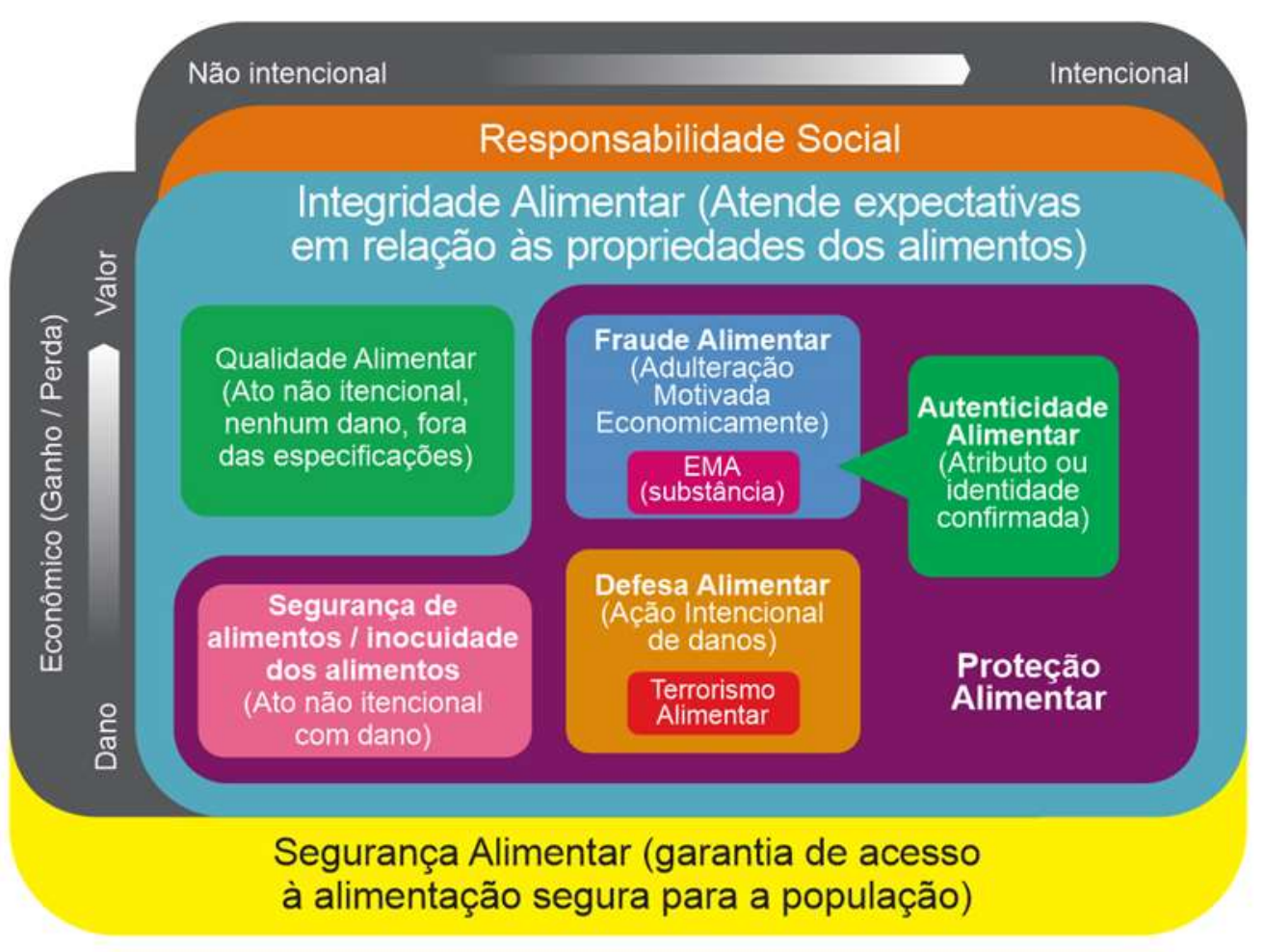

Fonte: Adaptado de Spink (2019).

Estes conceitos, embora relacionados tal como se apresentam na Figura 2, devem ser abordados de forma individual e integrada, abrangendo todas as vertentes analisadas, contemplando perigos de adulteração intencional, não intencional e acidental com a finalidade de proteção dos alimentos.

\section{Normas, metodologias e sistemas para estabelecer food defense}

Os objetivos do conceito food defense envolvem: reduzir a probabilidade de ocorrer um ataque malicioso, reduzir ou minimizar os impactos que possam advir, proteger a reputação organizacional, produtos e instalações, população em geral, bem como demonstrar aos consumidores que a organização adota procedimentos preventivos para proteger os alimentos fornecidos (Guide to Developing a Food Defense Plan for Food Processing Plants, 2008).

Existe uma série de sistemas para a implantação do food defense adequados às necessidades das organizações, desenvolvidos para avaliações de vulnerabilidades, de riscos e de ameaças associadas a práticas de contaminação, adulteração e fraude ao longo da cadeia alimentar, que correspondem as seguintes ferramentas: CARVER+ SHOCK, VACCP (Vulnerability Assessment and Critical Control Points), TACCP (Threat Assessment Critical Control Points), HACCDP (Hazard Analysis Critical Control and Defense Points), HARPC (Hazard Analysis and Risk-Based Preventive Controls) e ORM (Operational Risk Management). No entanto, estes sistemas devem ser inseridos e adaptados às organizações, que em sua grande maioria, já possuem seus próprios sistemas de segurança alimentar, através de padrões e normas internacionais implementados, tornando-se um grande desafio incorporar o conceito food defense às metodologias e normas da segurança alimentar, tais como: BRC Food, IFS Food e FSSC 22000 (Severino \& Almeida, 2016).

Existe, no entanto, uma lacuna de conhecimento em relação aos tipos de ameaças que se enquadram no escopo de uma estratégia de food defense e essas questões podem ser mitigadas e, sempre que possível, eliminadas (Food Safety System Certification 22000, 2019). As contaminações intencionais podem se dividir em duas categorias: a primeira (food defense) inclui as motivações de um indivíduo, um funcionário insatisfeito ou grupos ativistas, com finalidade de extorsão, danos e 
pressão política, utilizando o alimento como veículo de contaminação com a finalidade do terrorismo e a segunda (food fraud) inclui a substituição ou diluição de produtos alimentícios com ingredientes de menor valor, rótulo incorreto ou falsificado, com o intuito de fraudar e mascarar a verdadeira origem dos alimentos com a finalidade de ganhar acesso a mercados ou adquirir preços mais altos, são as causas mais comuns e denomina-se adulteração com motivação de ganho econômico, conhecido pela sigla em inglês EMA (Economically Motivated Adulteration). Embora, a finalidade das adulterações seja principalmente motivada pelo ganho econômico, é importante ressaltar que os dois casos de contaminação intencional resultam em efeitos prejudiciais para a saúde e a economia (Betancourt, 2017).

Em busca de auxiliar a indústria de alimentos, a FDA vem adequando as ferramentas mencionadas anteriormente, e em 2019 lançou a versão mais atualizada do Plan Builder, uma ferramenta tecnológica para avaliar as vulnerabilidades e apoiar na construção de um plano de food defense para evitar contaminações deliberadas em um sistema ou uma edificação. Facilita ao usuário pensar como o invasor, identificando os alvos mais atraentes ou mais propensos para um ataque, o que permite concentrar os recursos e planos de proteção nestes pontos (FDA, 2019).

Desse modo é importante que o Brasil como grande produtor e exportador conheça o conceito, metodologias e ferramentas que apoiam a implementação do food defense e se adeque às tendências do novo milênio.

\section{Regulamentações para food defense}

No início de 2011, após uma série de grandes incidentes de segurança alimentar, foi promulgada pela FDA a lei: Food Safety Modernization Act (FSMA), que visa reduzir o número de incidentes evitáveis de segurança alimentar nos EUA que podem impactar significativamente a saúde pública e que representam uma ameaça ao bem-estar econômico do sistema alimentar dos EUA, foi criada com a finalidade de proteger, mitigar, prevenir, detectar e responder frente a problemas de segurança intencional e terrorismo na cadeia produtiva. Esta lei, outorga à FDA o poder legislativo para regular qualquer fornecedor estrangeiro, entidade ou pessoa envolvida na elaboração de alimentos dos Estados Unidos (Food \& Drug Administration, 2016).

Os padrões de food safety mais significativos na Europa é o British Retail Consortium (BRC) e os International Featured Standards (IFS). De acordo com esses padrões, os requisitos de food defense são obrigatórios e incluem a implementação de análise de perigos, a avaliação de riscos relacionados e a identificação de áreas críticas (British Retailer Consortium, 2015).

Em maio de 2016, a FDA lançou a regra final da Lei de Modernização da Segurança Alimentar (FSMA- Food Safety Modernization Act) que visa impedir a adulteração intencional de atos destinados a causar danos em larga escala à saúde pública, incluindo atos de terrorismo direcionados ao suprimento de alimentos. Tais atos, embora não sejam prováveis, podem causar doenças, morte ou interrupção no suprimento de alimentos. Nesta regra a adulteração econômica é abordada nas regras finais de controles preventivos para alimentos de origem humana e animal (Food \& Drug Administration, 2016).

Em 2018 foi publicada a norma ISO 22000 (FSMS) que agora estabelece os requisitos para um sistema de gerenciamento de food defense. Ela mapeia o que uma organização precisa fazer para demonstrar sua capacidade de controlar os riscos à segurança de alimentos, a fim de garantir que os alimentos sejam seguros. Pode ser usada por qualquer organização, independentemente do seu tamanho ou posição na cadeia alimentar, da produção primária ao consumidor (ISO, 2018).

Se uma empresa de alimentos possui acordos de parceria ou exporta produtos para determinados países, é obrigada a aplicar os requisitos de food defense definidos pelas normas de segurança alimentar e pela legislação dos países com os quais a empresa se relaciona comercialmente. A food defense é uma preocupação em quase todas as áreas de negócios da indústria de alimentos, onde a contaminação intencional e a fraude alimentar podem representar uma série de ameaças aos consumidores e à saúde pública, além de prejudicar os negócios da empresa (Bogadi et al., 2016). 


\section{Implementação do plano de food defense}

Food defense envolve exigências que já são contempladas em um sistema de gestão food safety. Isso se refere à documentação do sistema de segurança de alimentos, rastreabilidade, documentação e registros do sistema de gerenciamento, medidas corretivas e preventivas, auditorias internas, treinamento de funcionários etc.

O FSSC 22000 da GFSI abrange a maior parte dos Requisitos da Lei de Modernização da Segurança Alimentar (FSMA), mas a questão da adulteração intencional do programa ainda precisa ser abordada. A Figura 3 descreve o Sistema de Gestão de Segurança de Alimentos, de acordo com a GFSI, onde os conceitos são muito semelhantes, segundo os quais a ameaça e a vulnerabilidade dos sistemas e instalações devem ser avaliadas usando os conceitos HACCP, só que de forma a evitar riscos de adulteração intencional. O GFSI definiu duas metodologias com esta finalidade: VACCP (vulnerabilidade / food fraud) e TACCP (ameaça / food defense) (GFSI, 2018b). Sendo TACCP (Threat Assessment Critical Control Point), avaliação de ameaças e pontos críticos de controle e VACCP (Vulnerability Assessment and Critical Control Point), avaliação de vulnerabilidades e pontos críticos de controle (Spink, 2019).

Figura 3. Sistema de Gestão de Segurança de Alimentos.

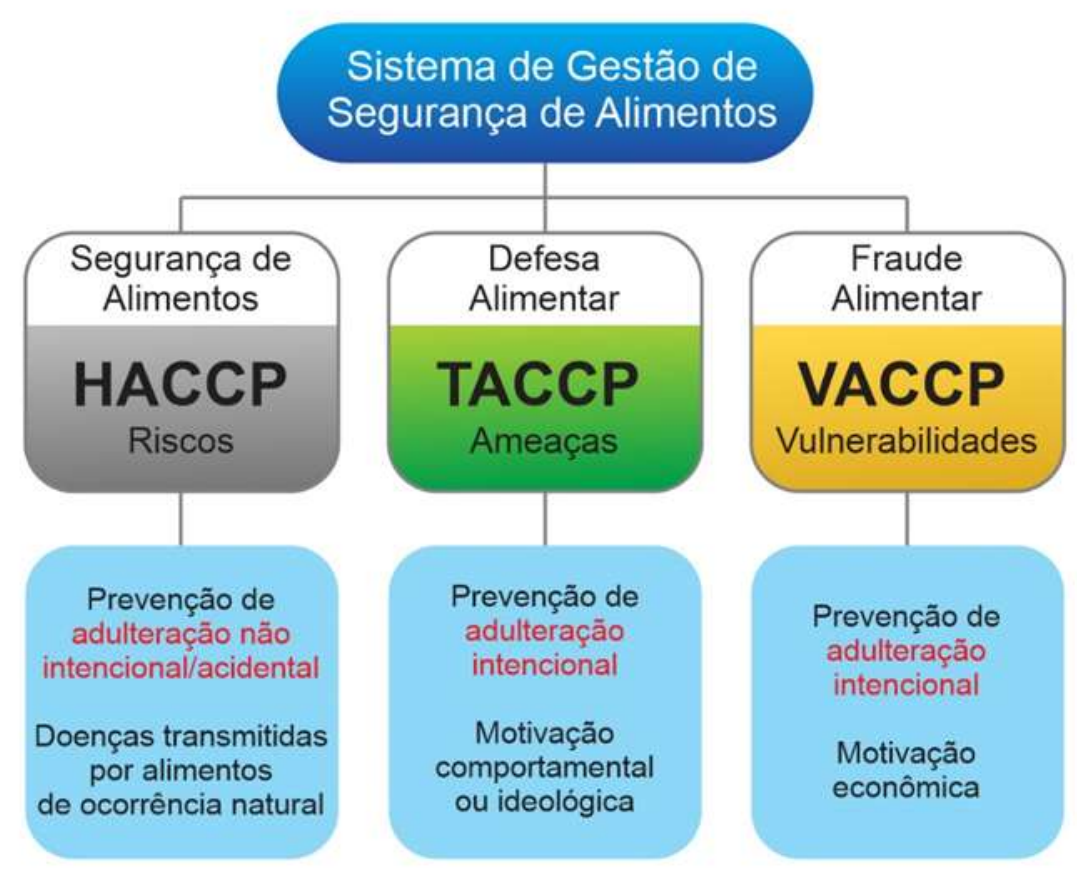

Fonte: Adaptado de FSSC 22000 (2019b).

A GFSI definiu o Food Safety Management System (FSMS) como um " guarda-chuva " demonstrado na Figura 3 que inclui HACCP (riscos / segurança de alimentos), TACCP (ameaças / defesa alimentar) e VACCP (vulnerabilidades / fraude alimentar).

As empresas de alimentos precisam decidir sobre a metodologia de gestão de riscos a ser utilizada. Existem eficientes métodos disponíveis e cada organização deve decidir pelo método que for mais aderente aos seus pré-requisitos. A avaliação de riscos é realizada com o objetivo de definir medidas de controle e preventivas (Dias et al., 2010).

\section{Processo de food defense, definido pela PAS 96}

Com a publicação da nova versão do PAS (Publicly Available Specification) 96:2017, o conteúdo cobre estratégias de gerenciamento de risco que podem ser adaptadas a operações de todos os tamanhos, em diferentes pontos da cadeia de 
suprimentos, fornecendo orientações sobre como as empresas podem detectar potenciais vulnerabilidades e as medidas que elas podem tomar para mitigá-las. Uma diferença em relação à versão anterior está nos anexos, que reconhecem abordagens complementares, como CARVER+Shock, EU 5-point action plan e o guia UK Food and Drink Federation. Além de informações e inteligência sobre os riscos emergentes para o fornecimento de alimentos, que podem ser usadas para atualizar dados de probabilidades de ataques (Food Safety Brazil, 2017).

De acordo com a PAS 96:2017 (PAS 96:2017 Guide to Protectingnand Defending Food and Drink from Deliberate Attack, 2017) para aplicar e gerenciar todas as atividades prescritas na área de food defense, uma equipe permanente do TACCP deve ser formada, o que pode incluir indivíduos com os seguintes conhecimentos: segurança, recursos humanos, tecnologia de alimentos, engenharia de processos, produção e operações, compras e suprimentos, distribuição e logística, tecnologia da informação, comunicações e comercial / marketing. A equipe do TACCP pode e deve modificar o processo do TACCP para melhor atender às suas necessidades e adaptá-lo a outras ameaças, conforme necessário, para lidar com quatro questões subjacentes: Quem pode querer atacar a empresa? Como podem fazer isso? Onde a empresa está vulnerável? Como detê-los? O processo TACCP concentra-se na adulteração e contaminação deliberadas.

\section{Processo de food defense, definido pela FDA}

Para implementar o plano de food defense, primeiro deve-se fazer a identificação das etapas do processo, seguida da implementação de estratégias de mitigação focadas para reduzir a probabilidade de contaminação intencional causada por um ato de terrorismo. O plano deve ser monitorado e todos os registros confidenciais sobre proteção alimentar e sobre adulteração intencional causada por atos de terrorismo, devem ser documentados. Uma etapa crucial para o sucesso do plano é o treinamento, que exige que as pessoas envolvidas nos processos com alimentos tomem consciência do conceito food defense e de suas responsabilidades (Office of the Federal Register, 2013).

A FDA (2020) também disponibilizou outros recursos para ajudar a indústria a identificar e mitigar possíveis vulnerabilidades para adulteração intencional, como: o programa ALERT, a ferramenta de treinamento Employees FIRST, uma base de dados Mitigations Strategies Database, um software Food Defense Plan Builder, um programa de exercícios emergenciais relacionados a alimentos (FREE-B), e manual de treinamento Food Defense 101.

\section{De nada adianta os modelos e ferramentas se não existir a cultura da segurança}

Atualmente, incidentes relacionados a contaminação de produtos alimentares é um enorme problema em toda a cadeia de suprimento de alimentos. Portanto, é necessário aumentar a conscientização da food defense a cada nível da cadeia alimentar, da fazenda ao garfo, das instituições governamentais e acadêmicas às redes de varejo, mídia e consumidores. A eficiência da empresa está na implementação de novos métodos, ferramentas e sistemas diretamente correlacionados com o nível de conhecimento e educação de seus funcionários. Uma nova implementação de um sistema de food defense em um país exige não apenas aumentar a conscientização dos funcionários no nível da empresa, mas também no nível das instituições governamentais (Bogadi et al., 2016).

Para que organismos institucionais e a sociedade possam exigir uma alimentação segura, é imprescindível que os conceitos, aspectos e fatores que se relacionam à segurança alimentar, sejam cada vez mais conhecidos e que façam parte da rotina na vida das pessoas. O conhecimento sobre os perigos biológicos, químicos e físicos que colocam os alimentos em risco, somado a consciência quanto ao impacto que a ação humana desordenada pode ter sobre a cadeia alimentar e sobre o meio ambiente, formam a base para a construção da cultura da segurança alimentar (Cabral \& Moura Neto, 2020).

Diante deste grande desafio de informar, educar e conscientizar sobre os aspectos de segurança alimentar, a GFSI lançou em 2015 um documento de referência, representado pela Figura 4, cujo objetivo é descrever as dimensões e o conteúdo 
crítico da segurança alimentar no contexto de uma cultura organizacional. O documento propõe cinco dimensões da Cultura de Segurança de Alimentos: Visão e Missão, Pessoas, Consistência, Adaptabilidade e Percepção de Perigos e Riscos (Figura 4) (GFSI, 2018a).

Figura 4. As cinco dimensões e os componentes críticos da Cultura de Segurança de Alimentos

\begin{tabular}{|c|c|c|c|c|}
\hline Visão e Missão & Pessoas & Consistência & Adaptabilidade & $\begin{array}{l}\text { Percepção de } \\
\text { Perigos } \\
\text { e Riscos }\end{array}$ \\
\hline $\begin{array}{c}\text { Estrutura } \\
\text { Organizacional, } \\
\text { Valores e } \\
\text { Propósito }\end{array}$ & $\begin{array}{l}\text { Governança } \\
\text { /Comunicação }\end{array}$ & $\begin{array}{c}\text { Senso de } \\
\text { Responsabilidade }\end{array}$ & $\begin{array}{l}\text { Expectativas de } \\
\text { Segurança de } \\
\text { Alimentos e } \\
\text { Estado Atual }\end{array}$ & $\begin{array}{c}\text { Informação e } \\
\text { Educação sobre } \\
\text { Riscos } \\
\text { Fundamentais }\end{array}$ \\
\hline $\begin{array}{l}\text { Definição de } \\
\text { Diretrizes e } \\
\text { Expectativas }\end{array}$ & $\begin{array}{c}\text { Organização do } \\
\text { Aprendizado }\end{array}$ & $\begin{array}{c}\text { Medição de } \\
\text { Desempenho }\end{array}$ & Agilidade & $\begin{array}{l}\text { Envolvimento } \\
\text { dos } \\
\text { Colaboradores }\end{array}$ \\
\hline $\begin{array}{c}\text { Liderança e } \\
\text { Comunicação }\end{array}$ & $\begin{array}{c}\text { Incentivos, } \\
\text { Recompensas e } \\
\text { Reconhecimento }\end{array}$ & Documentação & $\begin{array}{l}\text { Mudanças, } \\
\text { Gerenciamento } \\
\text { de Crises e } \\
\text { Solução de } \\
\text { Problemas }\end{array}$ & $\begin{array}{c}\text { Verificação } \\
\text { da Percepção de } \\
\text { Perigos e Riscos }\end{array}$ \\
\hline
\end{tabular}

Fonte: Adaptado de GFSI (2018 $)$.

\section{Considerações Finais}

O advento da pandemia COVID-19, novas pressões de alta de preços, economias em declínio e comércio global em constante mudança ao longo da cadeia de abastecimento do sistema alimentar, introduziram uma nova urgência para maior diligência na defesa de alimentos contra a contaminação deliberada por motivos econômicos ou terroristas. As iniciativas incluem o desenvolvimento de contramedidas específicas para minimizar ou eliminar vulnerabilidades, bem como o desenvolvimento de soluções práticas que aumentem a capacidade de identificar, conter, responder e recuperar-se rapidamente da contaminação intencional, real e ameaçada. Essas atividades devem abranger todo o sistema alimentar do campo à mesa em todo o mundo, desde os insumos até a venda no varejo, o consumidor, incluindo uma rápida resposta do sistema de saúde pública.

Food defense é um tema pouco pesquisado no Brasil e há informações limitadas de domínio público na área temática. Ao mesmo tempo existe uma lacuna de conhecimento inerente à indústria sobre quais estratégias de food defense precisam ser abordadas, há uma necessidade crescente das organizações da cadeia de abastecimento alimentar desenvolverem e adotarem o plano food defense para garantirem a entrada no mercado por meio de certificação de terceiros de seus sistemas de gestão.

De acordo com estudo feito por Praia e Henriques (2021), empresas de alimentos localizadas em Portugal, certificadas por pelo menos um padrão internacional que contemple food defense, ainda apresentam vulnerabilidades importantes a serem tratadas, e como principal proposta de estratégia de intervenção, as autoras enfatizam a formação em food defense sendo fundamental para facilitar a compreensão do conceito pelos gestores e pelos envolvidos nas indústrias de alimentos.

O mercado internacional impõe vários padrões, requisitos críticos e medidas de proteção, e as indústrias brasileiras para não perderem mercado ou até para expandirem sua capacidade de exportação, devem estar com planos de defesa bem implementados para assegurar a proteção dos alimentos quanto à contaminação intencional e maliciosa, sabotagem, 
bioterrorismo e outros pontos de vulnerabilidade às organizações, forçando as indústrias brasileiras a se enquadrarem rapidamente às legislações.

A partir desta pesquisa exploratória, surgem sugestões de trabalhos futuros, como estudos em setores específicos, ou em empresas que tenham food defense implantado e reconhecido por órgãos certificadores, com diversos objetivos: verificar a consistência do plano food defense, mensurar níveis de vulnerabilidades, mensurar níveis de cultura de segurança nas empresas, analisar quais setores que mais são cobrados por certificações food defense.

Espera-se que os resultados deste estudo despertem e orientem a adoção de procedimentos e medidas nos âmbitos conceitual e prático para prevenir e controlar a contaminação intencional de alimentos, de forma a atender às constantes mudanças e exigências dos mercados consumidores e a adoção de normas e legislações vigentes pertinentes à qualidade e segurança dos alimentos, ressaltando ainda a importância da disseminação dos conceitos de food defense através de treinamentos para capacitar e formar gestores e envolvidos aptos a implantarem de forma plena, os requisitos e o plano food defense, maximizando a redução das vulnerabilidades e garantindo um alimento seguro aos consumidores.

\section{Agradecimentos}

Este trabalho foi realizado com o apoio da Coordenação de Aperfeiçoamento de Pessoal de Nível Superior (CAPES Código de Financiamento 001), Fundação de Amparo à Pesquisa do Estado do Rio de Janeiro (FAPERJ, E-26.202.749/2018) e Conselho Nacional de Desenvolvimento Científico e Tecnológico (CNPq, 311936/2018-374 0).

\section{Referências}

Agriculture, U. S. D. of. (2021). Food Safety and Inspection Service. https://www.fsis.usda.gov/food-safety/food-defense-and-emergency-response/fooddefense

Albrecht, R., \& Ohira, L. (2000). Bases de dados: metodologia para seleção e coleta de documentos. Revista ACB: Biblioteconomia Em Santa Catarina, 5(5), $131-144$.

Baldy, J. L. S. (1991). Introdução ao Estudo das Doenças Transmissíveis. Conceitos básicos. (Sarvier (ed.)).

Betancourt, R. S. (2017). Defesa alimentar: avaliação e aplicação da ferramenta CARVER+Shock na indústria do leite no Brasil. Universidade de São Paulo.

Bogadi, N. P., Banović, M., \& Babić, I. (2016). Food defence system in food industry: perspective of the EU countries. Journal Fur Verbraucherschutz Und Lebensmittelsicherheit, 11(3), 217-226. https://doi.org/10.1007/s00003-016-1022-8

British Retailer Consortium. (2015). BRC - Global Standard Food Safety - Issue 7 Frequently Asked Questions. 7, 1-14. https://www.brcglobalstandards.com/media/63848/brc_global_standard_for_food_safety_issue_7_faqs-1.pdf

Cabral, É. S. G., \& Moura Neto, L. G. de. (2020). Segurança Alimentar à luz de novas ferramentas educativas na pedagogia crítica. Research, Society and Development, 9(8), e910986254. https://doi.org/10.33448/rsd-v9i8.6254

Dalziel, G. R. (2009). Food defence incidents 1950-2008: A Chronology And Analysis Of Incidents Involving The Malicious Contamination Of The Food Supply Chain.Technical Report. Centre of Excellence for National Security (CENS), 66. http://www.food-defense.it/1/upload/rsis_food_defence_170209.pdf

Davison, N., \& Neil. (2005). The Role of Scientific Discovery in the Establishment of the First Biological Weapons Programmes. EMBO Reports, 7(1), S45S49. https://doi.org/10.1038/sj.embor.7400689

Dias, J., Heredia, L., Ubarana, F., \& Lopes, E. (2010). Implementação de Sistemas da Qualidade e Segurança dos Alimentos (Vol. 01, pp. 1-160). Sociedade Brasileira de Ciência e Tecnologia de Alimentos.

Elliott, C., \& Goethem, V. (2018). Food Integrity Handbook Aguide to food authenticity issuesand analytical solutions (J.-F. Morin \& M. Lees (eds.)). Eurofins Analytics France. https://doi.org/https://doi.org/10.32741/fihb

F.F. Busta and S.P. Kennedy. (2011). Advances in Food Protection Focus on Food Safety and Defense. In Magdy Hefnawy (Ed.), NATO Science for Peae aned Security Series A - Chemistry and Biology. Springer. https://doi.org/10.1007/978-94-007-1100-6_7

FDA. (2019). Food Defense Plan Builder Version 2 . 0 User Guide. https://www.fda.gov

Food \& Drug Administration, U. S. (2016). FDA Food Safety Modernization Act.

Food Safety Brazil. (2017). Lançada a versão 2017 do Guia PAS 96, que trata de Food Defense. https://foodsafetybrazil.org/versao-2017-guia-pas-96-fooddefense/ 
Food Safety Department, W. (2008). Terrorist threats to food: guidance for establishing and strengthening prevention and response systems. American Journal of Agricultural Economics, 90, 1265-1271. https://doi.org/10.1111/j.1467-8276.2008.01215.x

FSSC 22000. (2019a). Esquema FSSC 22000 versão 5. www.fssc22000.com

FSSC 22000. (2019b). Food Safety System Certification 22000. [Online]. http://www.fssc22000.com/documents/home.xml?lang=en

Food Safety System Certification 22000, Food Safety System Certification 22000 - Guidance Document: Food Fraud Mitigation (2019). https://www.fssc22000.com/wp-content/uploads/19.0528-Guidance_Food-Defense_Version-5.pdf

GFSI. (2018a). Cultura de segurança de alimentos. GFSI, 1-59. https://mygfsi.com/news-and-resources/?type=publications\&topic=food-safety-culture\&lang

GFSI. (2018b). Tackling Food Fraud through Food Safety Management Systems. Gfsi, 1-10. https://www.mygfsi.com/files/Technical_Documents/201805food-fraud-technical-document-final.pdf

\section{GFSI BENCHMARKING REQUIREMENTS VERSION 2020, (2020).}

HM Government. (2014). Elliott review into the integrity and assurance of food supply Networks. In The National archives (Vol. 157, Issue March). https://www.gov.uk/government/publications/elliott-review-into-the-integrity-and-assurance-of-food-supply-networks-final-report

ISO. (2018). ISO 22000:2018 Food safety management systems - Requirements for any organization in the food chain. Internacional Organization for Standardization. https://www.iso.org/standard/65464.html

Klitzke, C. J. S. C. H. (2015). Food Defense Best Practices Reported by Public School. Journal of Child Nutrition and Management, 1-13.

Luna, E. J. A. (2002). A emergência das doenças emergentes e as doenças infecciosas emergentes e reemergentes no Brasil. Revista Brasileira de Epidemiologia, 5(3), 229-243. https://doi.org/10.1590/s1415-790x2002000300003

Office, D. H. S. (2007). Office of Inspector General The Department of Homeland Security's Role in Food Defense and Critical Infrastructure Protection February 2007. February.

Office of the Federal Register. (2013). Federal Register (Vol. 78, Issue 247). https://www.govinfo.gov/app/details/FR-2013-12-24/2013-30373/context

Pear, R. (2004, December 4). U.S. Health chief, stepping down, issues warning. The New York Times, 1.

Praia, E. F., \& Henriques, A. R. (2021). Assessing the implementation of food defense requirements in industrial meat-based food processors. Brazilian Journal of Food Technology, 24, 1-14. https://doi.org/10.1590/1981-6723.20120

Ribeiro-Furtini, L. L., \& Abreu, L. R. de. (2006). Utilização de APPCC na indústria de alimentos. Ciência e Agrotecnologia, 30(2), 358-363. https://doi.org/10.1590/s1413-70542006000200025

Rodrigues, W. C. (2007). Metodologia Científica. In FAETEC/IST (pp. 1-20).

Santos, C. S. dos, Shinohara, N. K. S., Macedo, I. M. E., \& Souza, M. D. L. C. de. (2020). Fraud in Food Produts. Research, Society and Development, 9(10), e3289108692. https://doi.org/10.33448/rsd-v9i10.8692

Schatzmayr, H. G. (2013). Bioterrorism and pathogenic microorganisms. Historia, Ciencias, Saude - Manguinhos, $20(4)$, 1735-1749. https://doi.org/10.1590/S0104-597020130005000016

Severino, P., \& Almeida, D. (2016). Food Defense. In Agrobook. Agrobook. https://doi.org/10.1002/9781119341178.ch13

Severino, P. R. de S. (2016). Food Defense e a sua relação com as normas IFS V6, BRG V7 e FSSC 22000. Universidade de Lisboa.

Spink, J., \& Moyer, D. C. (2011). Defining the Public Health Threat of Food Fraud. Journal of Food Science, 76(9). https://doi.org/10.1111/j.17503841.2011.02417.x

Spink, J. W. (2019). Food Fraud Prevention. In Food Fraud Prevention. https://doi.org/10.1007/978-1-4939-9621-6

PAS 96:2017 Guide to protectingnand defending food and drink from deliberate attack, BSI Standards Limited (2017). https://www.food.gov.uk/sites/default/files/media/document/pas962017_0.pdf

Guide to developing a food defense plan for food processing plants, (2008).

U.S. Food \& Drug Administration. (2020). Food Defense. Food \& Beverages. https://www.fda.gov/food/food-defense

U.S. Food and Drug Administration. (2007). Food Protection Plan An integrated strategy for protecting the nation's food supply. https://www.fda.gov/media/75264/download

World Health Organization. (2007). The world health report 2007: a safer future: global public health security in the 21st century. https://apps.who.int/iris/handle/10665/43713 